\title{
Microstructural Evolution in Materials during Thermal Processing
}

\author{
Joseph K. L. Lai, ${ }^{1}$ Brian Ralph, ${ }^{2}$ Zhiwen Chen, ${ }^{3}$ and Kin Ho Lo ${ }^{4}$ \\ ${ }^{1}$ Department of Physics and Materials Science, City University of Hong Kong, Tat Chee Avenue, Kowloon, Hong Kong \\ ${ }^{2}$ Brunel University, Kingston Lane, Uxbridge, UB8 3PH, UK \\ ${ }^{3}$ School of Environmental and Chemical Engineering, Shanghai University, Shangda Road 99, P.O. Box 144, Shanghai 200444, China \\ ${ }^{4}$ Department of Electromechanical Engineering, University of Macau, Avenida Padre Tomás Pereira, Taipa, Macau, China \\ Correspondence should be addressed to Joseph K. L. Lai, apjoelai@cityu.edu.hk
}

Received 16 October 2011; Accepted 16 October 2011

Copyright (c) 2012 Joseph K. L. Lai et al. This is an open access article distributed under the Creative Commons Attribution License, which permits unrestricted use, distribution, and reproduction in any medium, provided the original work is properly cited.

The study of materials science is mainly based on our understanding of the relationship between microstructure and materials properties. Factors such as temperature, stress, and environment can lead to microstructural evolution with a concomitant change in properties. It is the materials scientist's role to harness our knowledge on this relationship to produce materials with improved properties, or to predict the performance of existing materials under various service conditions.

Over the past two decades, there have been a burgeoning number of novel or improved materials. This development is accompanied by significant advances in techniques in materials characterization as well as increased sophistication in theoretical methods for the analysis of microstructures. The application of these new techniques and methodologies to study the microstructural evolution of novel materials offers tremendous opportunities for scientific advancement on many fronts, with the prospect of providing new solutions to problems which have hitherto remained intractable.

The papers published in this special issue encompass various materials systems: metals and alloys, glass ceramics, thin films, nanowire, and oxide powders for engineering applications.

The paper by Dehmas et al. reports an investigation on the precipitate phases in Inconel 718, an important alloy for high temperature applications such as turbine blades. Using transmission electron microscopy and X-ray diffraction, the authors were able to confirm the presence of rotationordered domains in $\delta$ plates and explain unexpected contrast by double diffraction due to overlapping phases.
The paper by Ukai et al. is also focused on Nickel-based super-alloy for high temperature turbine applications. This study has enhanced our understanding of the precipitation and growth kinetics of $\gamma^{\prime}$ and its characteristics in mechanically alloyed materials.

The paper by Hiraoka et al. reports the results of an investigation on the strength and ductility of tungsten sheets after various recrystallization treatments. Three-point bend tests were performed at temperatures between 290 and $500 \mathrm{~K}$. Fracture surfaces were examined by scanning electron microscopy. The mechanical properties of tungsten and molybdenum were compared.

A glass ceramic material was studied by Montedo et al. in the their paper. The material studied is a candidate material for applications such as laminated tapes for screenprinted electronic components. The crystallization kinetics was studied using differential thermal analysis and scanning electron microscopy.

Other two papers were written by the same team of authors. Two titanium oxide glass ceramic materials with $\mathrm{La}_{2} \mathrm{O}_{3}$ addition, one strontium rich and one lead rich, were studied. The crystallization behavior of these materials was investigated using differential thermal analysis and X-ray diffraction.

Microstructural evolution during rapid thermal annealing of Ge nanowires was investigated by Tang et al. in the seventh paper. Scanning electron microscopy and transmission electron microscopy were used to observe $\mathrm{Ni}_{2} \mathrm{Ge} / \mathrm{Ge} / \mathrm{Ni}_{2} \mathrm{Ge}$ nanowire heterostructures. This work has 
important implications for the future development of field effect transistors.

Wang et al. present their work on zinc oxide thin films in the their paper. Two types of thin films were investigated: Ag ion-exchanged soda-lime glasses and aluminium-doped $\mathrm{ZnO}(\mathrm{AZO})$. Changes in chemical states and structural modification during and after heat treatment were explored. The results provide the basic information for determining the optimum heat treatment to grow silver quantum dots of various size and density in thin films.

Another interesting contribution on the subject of $\mathrm{ZnO}$ thin films appears in one of the papers. Japanese researchers from the Kochi University of Technology have developed a new technique for measuring the Youngs modulus of ultrathin films with thickness in the range of about $10 \mathrm{~nm}$. The technique was applied to study the mechanical properties of $\mathrm{Ga}$-doped $\mathrm{ZnO}$ thin films, a possible substitute material for indium-tin-oxide transparent electrodes.

Another paper deals with powder materials for Lithium ion battery applications. Akao et al. from the University of Fukui produced $\mathrm{LiNi}_{0.5} \mathrm{Mn}_{1.5} \mathrm{O}_{4}$ cathode materials by flame combustion using a spray pyrolysis apparatus. Xray diffraction revealed that, after calcination at $900^{\circ} \mathrm{C}$, crystalline powders with polygonal morphology and narrow particle size distribution were obtained.

The paper by Sola et al. is concerned with a literally "down to earth" problem. For many years, scientists have been developing ways to make use of fly ash in order to turn it from an industrial waste with environmental and storage problems into a useful material. The most obvious solution to this conundrum is to use it as an additive in sintered briquettes in the construction industry. These authors have determined the effect of different types of fly ash on the compressive strength of sintered briquettes. They showed that sintered briquettes samples made with Tuncbilek fly ash had higher percentage of glassy phase and compressive strength.

Papers published in this special issue will be of interest to materials scientists and engineers who wish to extend their knowledge on the effect of thermal processing on a broad range of materials. We would like to take this opportunity to thank all the authors who have submitted their papers to this publication. 

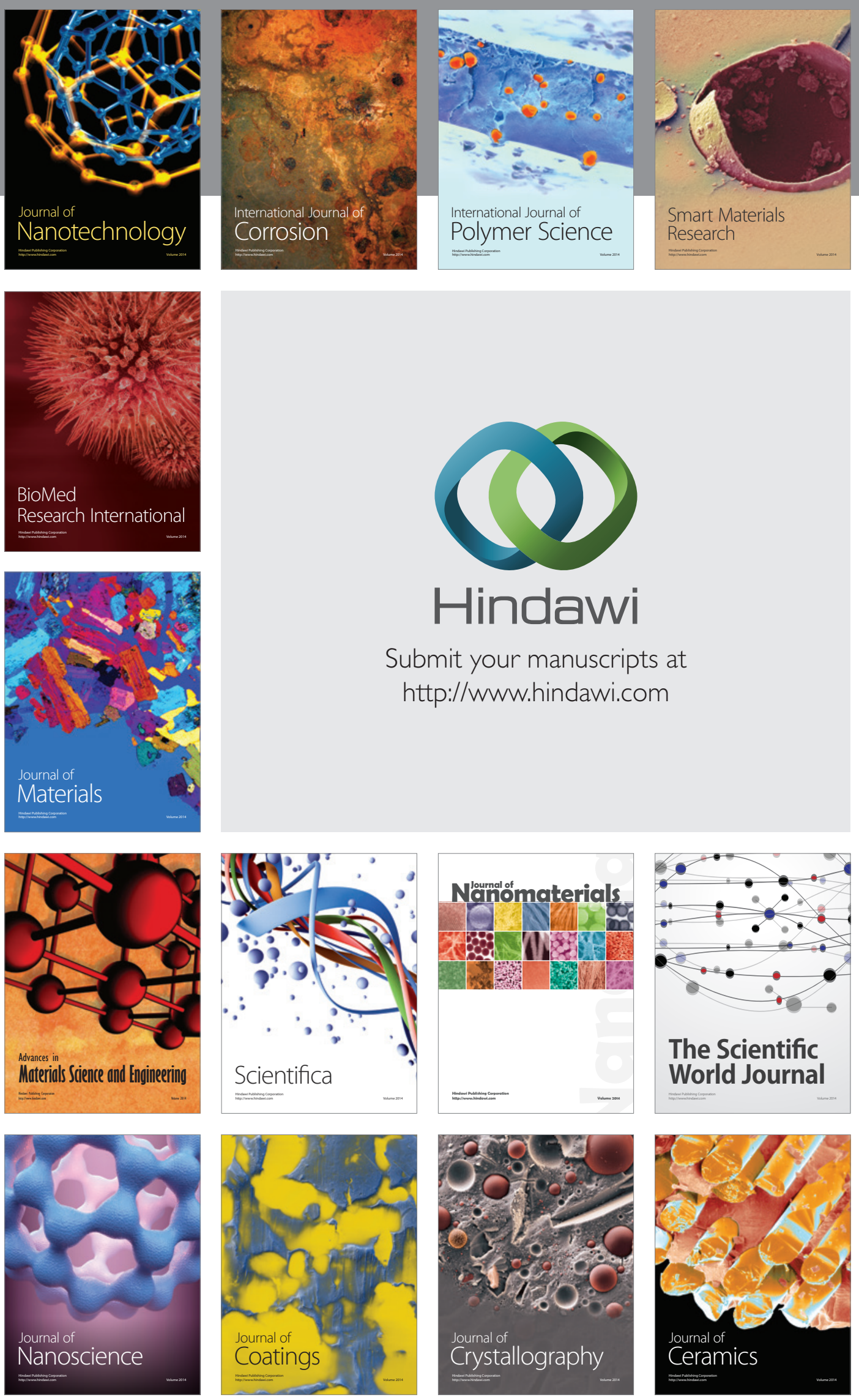

The Scientific World Journal

Submit your manuscripts at

http://www.hindawi.com

\section{World Journal}

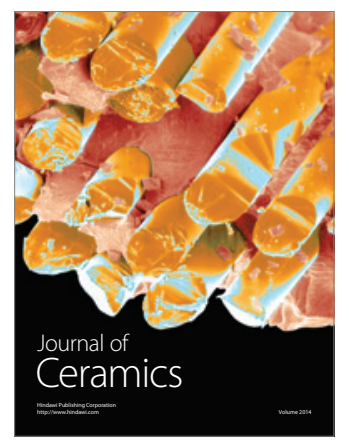

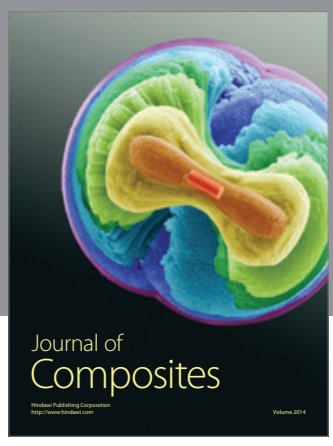
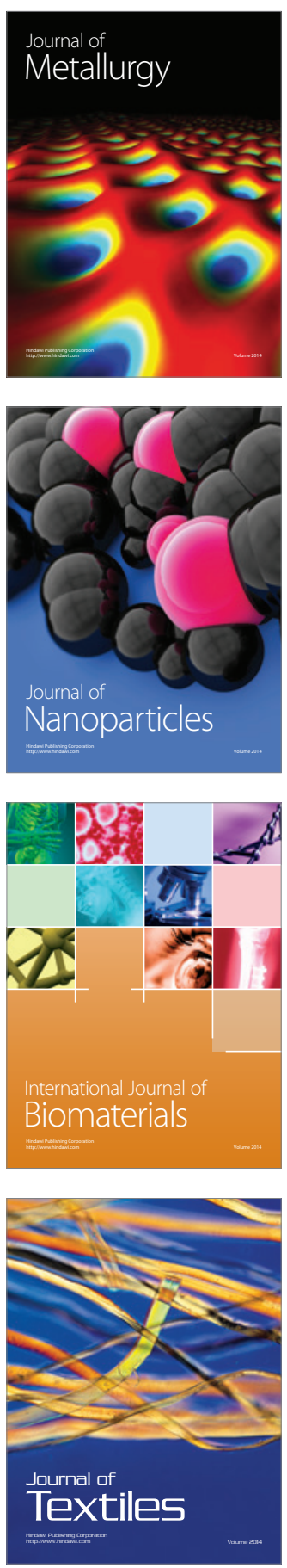